Proceedings of the 3rd International Congress APMAS2013, April 24-28, 2013, Antalya, Turkey

\title{
Synthesis, Structural and Optical Properties of Mo-Doped ZnO Thin Films
}

\begin{abstract}
A. MEKKI* AND N. TABET
Physics Department, King Fahd University of Petroleum and Minerals, Dhahran 31261, Saudi Arabia

Transparent zinc oxide thin films doped with molybdenum have been prepared using a DC reactive magnetron sputtering on glass substrates from metallic ZnMo target. The three films, called $A, B$, and $C$, had 1.9 at.\% Mo, 2.8 at.\% Mo and 4.7 at.\% Mo, respectively. The composition of the films was determined by X-ray photoelectron spectroscopy. The analysis of Mo $3 d_{5 / 2}$ core level spectra indicated that Mo exist in the films in Mo ${ }^{6+}$ oxidation state irrespective of the Mo content in the film. The X-ray diffraction spectrum of film $A$ showed a texture along the 002 orientation, while that of film $B$ showed two peaks, one at $\theta \approx 34.5^{\circ}$ and the other at $\approx 36.5^{\circ}$ corresponding to 002 and 101 orientations, respectively. Film $C$ showed two small peaks corresponding to 100 and 110 orientations. Optical measurements showed that all three films had a transmittance of about $80 \%$. The energy band gap showed a linear increase as Mo concentration increases from $3.29 \mathrm{eV}$ to $3.38 \mathrm{eV}$. The atomic force microscopy image of film $A$ showed a homogeneous morphology of the surface of the film, while the atomic force microscopy images of films $B$ and $C$ showed an inhomogeneous one.
\end{abstract}

DOI: 10.12693/APhysPolA.125.365

PACS: 81.15.Aa, 78.20.-e, 61.05.cp, 61.05.cj

\section{Introduction}

Zinc oxide $(\mathrm{ZnO})$ has attracted a considerable number of researchers over the last two decades because of the wide range of applications [1]. The development of nanotechnology induced an increasing interest for nanostructured semiconductors and $\mathrm{ZnO}$ appears to be a remarkable oxide that can be shaped in a vast variety of nanostructures such as nanorods [2], nanobelts [3], nanosheets [4], and nanospheres [5].

There have been many reports on the fabrication of transition-metal-doped $\mathrm{ZnO}$ bulk and thin film specimens. For example, studies have been reported on Ti-doped [6], $V$-doped [7], Cr-doped [8], Mn-doped [9], Fe-doped [10], Co-doped [11], Ni-doped [12], and Cu-doped [13] $\mathrm{ZnO}$.

In a recent study [14], Mo-doped zinc oxide thin films with high transparency and relatively low resistivity was prepared by RF magnetron sputtering at room temperature under different Ar pressure. The films had hexagonal structure and a preferred orientation along the $c$-axis. The resistivity increased as Ar pressure increased. The lowest resistivity achieved was $9.2 \times 10^{-4} \Omega \mathrm{cm}$ for the samples deposited at Ar pressure of $0.6 \mathrm{~Pa}$ with a Hall mobility of $30 \mathrm{~cm}^{2} \mathrm{~V}^{-1} \mathrm{~s}^{-1}$ and a carrier concentration of $2.3 \times 10^{20} \mathrm{~cm}^{-3}$. The average transmittance in the visible range exceeded $88 \%$ for all the samples. The optical band gap decreased from 3.27 to $3.15 \mathrm{eV}$ with increasing Ar pressure. In another study [15] Mo-doped ZnO transparent conductive thin films were prepared by dc reactive magnetron.

\footnotetext{
*corresponding author; e-mail: akmekki@kfupm.edu.sa
}

The structure, surface morphology, chemical state, optical and electrical properties of ZnMo films were studied. The films were polycrystalline with the hexagonal crystal structure, and the surface morphology measured by AFM demonstrated that the surface was smooth and compact. Chemical state analysis revealed that molybdenum atoms existed mainly in $\mathrm{Mo}^{6+}$ and $\mathrm{Mo}^{5+}$ ions. The energy bands increased from $3.37 \mathrm{eV}$ to $3.8 \mathrm{eV}$ with the increase in carrier concentrations.

In this work, we report on the synthesis of Mo doped $\mathrm{ZnO}$ thin films obtained by $\mathrm{DC}$ reactive magnetron sputtering technique. The objective is to investigate the effect of Mo doping on the optoelectronic properties of $\mathrm{ZnO}$ thin films. In addition, X-ray photoelectron spectroscopy (XPS) was used to determine the actual oxidation state of Mo in the films in order to confirm some published results which suggested the presence of both $5+$ and $6+$ oxidation states of $\mathrm{Mo}$ atoms in $\mathrm{ZnO}$ lattice [15].

\section{Experimental procedure}

Mo doped $\mathrm{ZnO}$ thin films were deposited by DC reactive magnetron sputtering on ordinary optical microscope glass slides. A disc of pure (99.999\%) metallic Zn was used as a target. The target was covered by a varying number of high purity (99.99\%) Mo wires starting with one wire and adding each time more wires to obtain films with different Mo concentrations. The XPS spectra were recorded using an Electron Spectrometer of the type VG-ESCALAB MKII. The structure of the thin films was studied using Shimadzu XRD-6000 X-ray diffractometer using a $\mathrm{Cu}$ anode. The microstructure and surface morphology was studied by atomic force microscopy (AFM) using INNOVA-Veeco AFM system in tapping mode. The optical transmittance of the films was measured between $200 \mathrm{~nm}$ and $800 \mathrm{~nm}$ using a JASCO V-570 Spectrophotometer. 


\section{Results and discussion}

Figure 1 shows the XRD patterns of the $\mathrm{ZnO}$ thin films with different Mo doping. It is clear from the figure that for sample $A$ (1.9 at.\% Mo), a single peak of $\mathrm{ZnO}(002)$ is observed at $2 \theta=34.36^{\circ}$. The $2 \theta$ value is close by less than that of the standard $\mathrm{ZnO}$ crystal $\left(34.45^{\circ}\right)$. For sample $B$ (2.8 at.\% Mo), three peaks of $\mathrm{ZnO}(100), \mathrm{ZnO}$ (002), and $\mathrm{ZnO}$ (101) are observed. The intensity of the 002 peak is much more that that of the 101 peak, while the intensity of the 100 peak is very small. For sample $C$ (4.7 at.\% Mo), the 002 and 101 peaks almost disappear while the 100 peak becomes stronger and a new peak (110) at $2 \theta=56.13^{\circ}$ appears. The position of 002 peak for sample $B$ is $2 \theta=34.24^{\circ}$. This value is less that of $\mathrm{ZnO}$ crystal and less than that of sample A. A decrease in $2 \theta$ for the 002 peak with increase in Mo doping might indicate an increase in the $c$ lattice parameter. The grain size can be estimated from the XRD diffraction peaks using Scherrer's formula. The (002) peak was used to estimate the grain size for samples $A$ and $B$, since it is the most intense peak, while the (100) peak was used for sample $C$. The Scherrer formula gave an estimated grain size of about $17 \mathrm{~nm}, 34 \mathrm{~nm}$, and $23 \mathrm{~nm}$ for films $A, B$, and $C$, respectively. The chemical states of oxygen and molybdenum atoms in these films were examined by XPS.

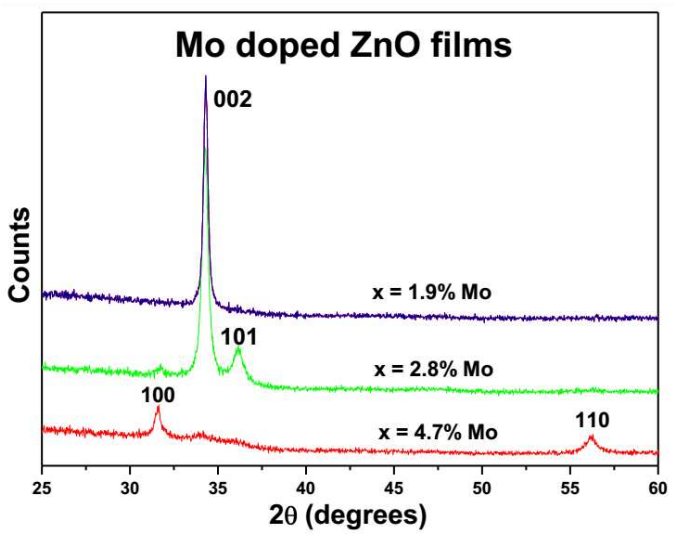

Fig. 1. XRD spectra of three $\mathrm{ZnO}$ films doped with Mo showing the change in structure of the film with Mo concentration.

The $\mathrm{O} 1 s$ and Mo $3 d$ XPS spectra of the as-deposited films are shown in Figs. 2a and 3a, respectively. The $\mathrm{O} 1 \mathrm{~s}$ core level peaks shown in Fig. 2a have a binding energy (BE) of about $530.2 \mathrm{eV}$ for all three films. There is an asymmetry on the higher BE side of the main peak due to moisture (hydroxide group). The $\mathrm{O} 1 s$ peaks were all fitted with two contributions. Figure $2 \mathrm{~b}$ shows the fitting of the $\mathrm{O} 1 s$ of film $A$ to two peaks, one at a BE of $530.2 \mathrm{eV}$ with full width at half maximum (FWHM) of $2.5 \mathrm{eV}$ due to oxygen in the $\mathrm{ZnO}$ structure and the other at a BE of $531.7 \mathrm{eV}$ with a FWHM of $2.6 \mathrm{eV}$ due to oxygen in hydroxide environment. The Mo $3 d_{5 / 2}$ peak is symmetric and has a $\mathrm{BE}$ of about $232.5 \mathrm{eV}$ for all three films. The standard values of the $\mathrm{BE}$ of $\mathrm{Mo} 3 d_{5 / 2}$ for
$\mathrm{Mo}^{6+}, \mathrm{Mo}^{5+}, \mathrm{Mo}^{4+}, \mathrm{Mo}^{3+}$ and metallic Mo are $232.6 \mathrm{eV}$, $231.5 \mathrm{eV}, 230.6 \mathrm{eV}, 230 \mathrm{eV}$, and $228 \mathrm{eV}[16,17]$.
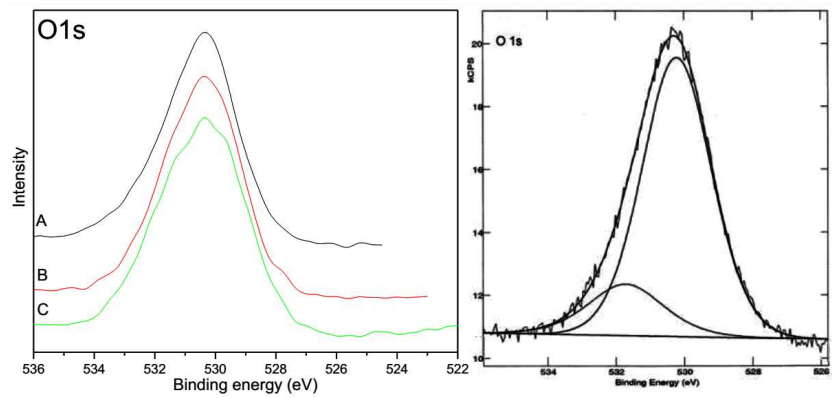

Fig. 2. (a) Core level XPS spectra of the three films, (b) XPS for the O $1 s$ spectrum of $x=2.8 \%$ Mo film fitted with two contributions.

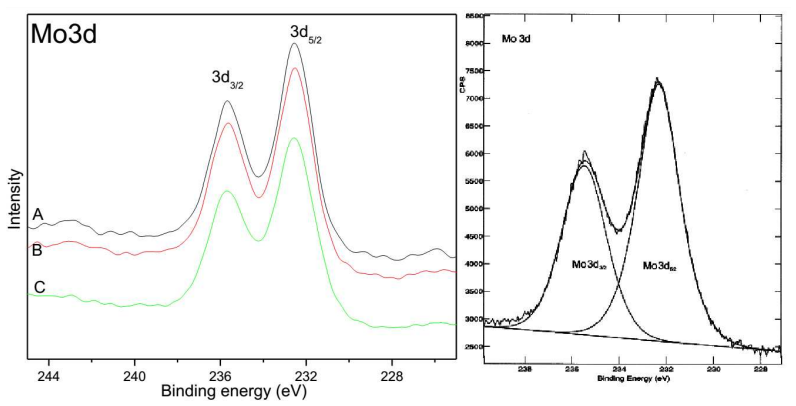

Fig. 3. (a) Core level spectra of the three films, (b) XPS for Mo $3 d$ spectrum of $x=2.8 \%$ Mo film showing the spin-orbit doublet.

It can be noted from Fig. 3a that the Mo $3 d$ spectra of the three films are symmetric and do no show any shoulder. We curve fitted the Mo $3 d$ spectrum for film $A$ as shown in Fig. $3 \mathrm{~b}$ for film $A$. It is clear from the figure that a single narrow doublet peak fits well the spectrum. The $\mathrm{BE}$ of the fitted Mo $3 d_{5 / 2}$ is $232.5 \mathrm{eV}$ with a FWHM of $2.2 \mathrm{eV}$ in agreement with previous work [17]. We conclude that Mo ions in our films are in the $\mathrm{Mo}^{6+}$ oxidation state forming $\mathrm{MoO}_{3}$ structure. No lower oxidation states were detected by XPS in these films. This result may be related to the pure oxygen atmosphere that was used in this work while the partial pressure of oxygen was set to $5 \times 10^{-2} \mathrm{~Pa}$ to $1.5 \times 10^{-1} \mathrm{~Pa}$.

The transmittance spectra in the wavelength range 200-2000 nm for the three films with different Mo doping level are shown in Fig. 4a. The average transmittance is about $80 \%$ for all samples indicating the good transparency of the films in the visible region. Changes in Mo content in the films did not affect the transmittance. However, there is a slight shift in the transmittance curves which indicate changes in the band gap energy $E_{\mathrm{g}}$. Figure $4 \mathrm{~b}$ (inset) shows a quasi linear variation of the energy gap as a function of Mo doping within the explored doping range. 

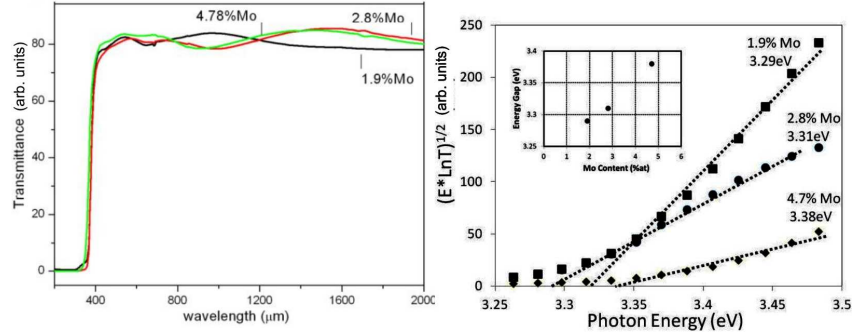

Fig. 4. (a) Transmittance spectra for the three Mo doped $\mathrm{ZnO}$ films and (b) $\left(E^{*} \ln T\right)^{2}$ vs. photon energy and energy gap vs. Mo content (inset).

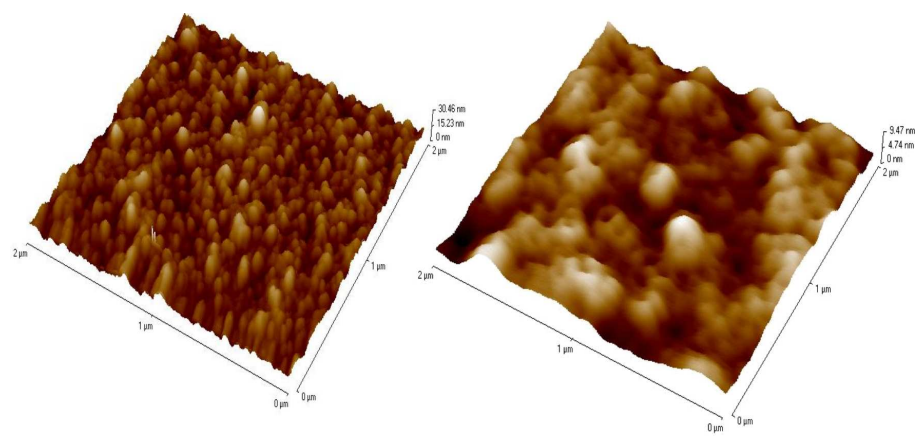

Fig. 5. AFM image of (a) 1.9\% Mo doped film showing a single [002] orientation of the grains and (b) $2.8 \% \mathrm{Mo}$ doped film with less pronounced texture.

The AFM images of the Mo doped thin films are shown in Fig. 5 in a $2 \mu \mathrm{m} \times 2 \mu \mathrm{m}$ region of the film. The AFM image of film $A$ shows in Fig. 5a grains with small size of the order of $10 \mathrm{~nm}$ and a well defined unidirectional orientation (002) in agreement with the XRD pattern of this film. The AFM images of film $B$ shown in Fig. 5b, indicate non-uniform films with non-well-defined grain having various orientations and much bigger size. The AFM image of film $C$, not shown here, shows a non-uniform film and probably with amorphous regions. These results are in agreement with the XRD findings.

\section{Conclusion}

Polycrystalline Mo doped $\mathrm{ZnO}$ thin films of various textures were deposited on glass substrate by DC reactive magnetron sputtering. With Mo doping the XRD pattern shows a texture change from a preferential orientation along [002] direction to [100] and [110] and an increase in grain size. The XPS data indicate that Mo is in the $6+$ oxidation state irrespective of the Mo doping. The optical measurements indicate an $80 \%$ transmittance and an increase in band gap with increase in Mo content. The AFM images of the low Mo content indicate a uniform film, a 002 grains orientation and about $15 \mathrm{~nm}$ grain sizes.

\section{Acknowledgments}

The authors would like to acknowledge the support of KFUPM and the Physics Department at KFUPM.

\section{References}

[1] Z.L. Wang, J. Phys., Condens. Matter 16, R829 (2004).

[2] H.S. Bae, M.H. Yoon, J.H. Kim, S. Im, Appl. Phys. Lett. 83, 5313 (2003).

[3] Y. Yang, W. Guo, J. Qi, Y. Zhang, Appl. Phys. Lett. 97, 223107 (2010).

[4] X. Hu, Y. Masuda, T. Ohji, K. Kato, J. Am. Ceram. Soc. 93, 881 (2010).

[5] R. Yogamalar, S. Anitha, R. Srinivasan, A. Vinu, K. Ariga, A.C. Bose, J. Nanosci. Nanotechnol. 9, 5966 (2009).

[6] Y.R. Park, K.J. Kim, Solid State Commun. 123, 147 (2003).

[7] H. Tabata, M. Saeki, S.L. Guo, J.H. Choi, T. Kawai, Physica B 308, 993 (2001).

[8] I. Satoh, T. Kobayashi, Appl. Surf. Sci. 216, 603 (2003).

[9] T. Fukumura, Z. Jin, M. Kawasaki, T. Shono, T. Hasegawa, S. Koshihara, H. Koinuma, Appl. Phys. Lett. 78, 958 (2001).

[10] S. Kolesnik, B. Dabrowski, J.J. Mais, J. Appl. Phys. 95, 2582 (2004).

[11] W. Prellier, A. Fouchet, C. Simon, B. Mercy, Mater Sci. Eng. B 109, 192 (2004).

[12] D.A. Schwartz, N.S. Norberg, Q.P. Nguyen, J.M. Parker, D.R. Gamelin, J. Am. Chem. Soc. 125, 13205 (2003).

[13] F. Leiter, H. Zhou, F. Henecker, A. Hofstaetter, D.M. Hofmann, B.K. Meyer, Physica B 308, 908 (2001).

[14] X. Xiu, Z. Pang, M. Lv, Y. Dai, L. Ye, S. Han, Appl. Surf. Sci. 253, 3345 (2007).

[15] C. Wu, J. Shen, J. Ma, S. Wang, Z. Zhang, X. Yang, Semicond. Sci. Technol. 24, 125012 (2009).

[16] C.D. Wagner, W.M. Riggs. L.E. Davis, J.F. Moulder, G.E. Muilenberg, Handbook of X-ray Photoelectron Spectroscopy, Physical Electronics Division, PerkinElmer, Eden Prairie, Minnesota 1979.

[17] K. Hada, J. Tanabe, S. Omi, M. Toshi, M. Nagai, J. Catal. 207, 10 (2002). 